\title{
THE PRIMITIVE COHOMOLOGY LATTICE OF A COMPLETE INTERSECTION
}

\author{
ARNAUD BEAUVILLE
}

\begin{abstract}
We describe the primitive cohomology lattice of a smooth even-dimensional complete intersection in projective space.
\end{abstract}

\section{INTRODUCTION}

Let $X$ be a smooth complete intersection of degree $d$ and even dimension $n$ in projective space. We describe in this note the lattice structure of the primitive cohomology $H^{n}(X, \mathbf{Z})_{\mathrm{o}}$. Excluding the cubic surface and the intersection of two quadrics, we find

$H^{n}(X, \mathbf{Z})_{\circ}=A_{d-1} \stackrel{\perp}{\oplus} k E_{8}( \pm 1) \stackrel{\perp}{\oplus} \ell U \quad$ or $\langle-d\rangle \stackrel{\perp}{\oplus} k^{\prime} E_{8}( \pm 1) \stackrel{\perp}{\oplus} \ell^{\prime} U$ where the numbers $k, \ell, k^{\prime}, \ell^{\prime}$ and the sign attributed to $E_{8}$ depend on the multidegree and dimension of $X$ - see Theorem 2.3 for a precise statement. The proof is an easy consequence of classical facts on unimodular lattices together with the Hirzebruch formula for the Hodge numbers of $X$.

We warn the reader that there are many ways to write an indefinite lattice as an orthogonal sum of indecomposable ones; for instance, when $8 \mid d$, both decompositions above hold. Still it might be useful to have a (semi-) uniform expression for this lattice. Related results, with a different point of view, appear in [L-W].

\section{Unimodular LATtices}

We will use the following standard notations for lattices: $U$ denotes the hyperbolic plane, and $\langle d\rangle$ the lattice $\mathbf{Z} e$ with $e^{2}=d$. If $L$ is a lattice, $L(-1)$ denotes the $\mathbf{Z}$-module $L$ with the form $x \mapsto-x^{2}$; if $n$ is a negative number, we put $n L:=|n| L(-1)$.

Date: December 20, 2018. 
Let $L$ be an odd unimodular lattice. A primitive vector $h \in L$ is said to be characteristic if $h \cdot x \equiv x^{2}$ (mod. 2$)$ for all $x \in L$; this is equivalent to saying that the orthogonal lattice $h^{\perp}$ is even ([L-W], Lemma 3.3).

Proposition 1.1. Let $L$ be a unimodular lattice, of signature $\left(b^{+}, b^{-}\right)$, with $b^{+}, b^{-} \geq 2$; put $s:=b^{+}-b^{-}, t=\min \left(b^{+}, b^{-}\right), u=\min \left(b^{-}, b^{+}-d\right)$. Let $h$ be a primitive vector in $L$ of square $d>0$, such that $h^{\perp}$ is even.

1) If $L$ is even or $8 \mid d$ we have $h^{\perp}=\langle-d\rangle \stackrel{\perp}{\oplus} \frac{s}{8} E_{8} \stackrel{\perp}{\oplus}(t-1) U$.

2) If $L$ is odd and $d \leq b^{+}$, we have $h^{\perp}=A_{d-1} \stackrel{\perp}{\oplus} \frac{s-d}{8} E_{8} \stackrel{\perp}{\oplus} u U$.

Proof : A classical result of Wall [W] tells us that $h$ is equivalent under $O(L)$ to any primitive vector $v$ of square $d$, provided $v$ is characteristic if so is $h$. If $L$ is even, we choose a hyperbolic plane $U \subset L$ with a hyperbolic basis $(e, f)$, and we put $v=e+\frac{d}{2} f$; then $v^{\perp}=\mathbf{Z}\left(e-\frac{d}{2} f\right) \stackrel{\perp}{\oplus} U^{\perp}$, and $U^{\perp}$ is an indefinite unimodular lattice, hence of the form $p E_{8}( \pm 1)$ $\stackrel{\perp}{\oplus} q$. Computing $b^{+}$and $b^{-}$we find the above expressions for $p$ and $q$.

Consider now the case when $L$ is odd. We first observe that since $h$ is characteristic, we have $d=h^{2} \equiv s(\bmod .8)([\mathrm{S}], \mathrm{V}$, Th. 2). Let

$$
L^{\prime}:=\left(\oplus_{i \leq d}^{\perp} \mathbf{Z} e_{i}\right) \stackrel{\perp}{\oplus} \frac{s-d}{8} E_{8} \stackrel{\perp}{\oplus} u U \quad \text { with } \quad e_{1}^{2}=\ldots=e_{d}^{2}=1 .
$$

$L^{\prime}$ is odd, indefinite and has the same signature as $L$, hence is isometric to $L$. We put $v=e_{1}+\ldots+e_{d}$. The orthogonal of $v$ in $\stackrel{\perp}{\oplus} \mathbf{Z} e_{i}$ is the root lattice $A_{d-1}$. By Wall's theorem $h^{\perp}$ is isometric to $v^{\perp}=A_{d-1} \stackrel{\perp}{\oplus}$ $\frac{s-d}{8} E_{8} \stackrel{\perp}{\oplus} u U$.

Suppose moreover that 8 divides $d$, so that $8 \mid s$. Then $L$ is isomorphic to $\mathbf{Z} e \stackrel{\perp}{\oplus} \mathbf{Z} f \stackrel{\perp}{\oplus} \frac{s}{8} E_{8} \stackrel{\perp}{\oplus}(t-1) U$, with $e^{2}=1, f^{2}=-1$. Taking $v=\left(\frac{d}{4}+1\right) e+\left(\frac{d}{4}-1\right) f$ gives the result.

\section{Complete intersections}

We will check that the hypotheses of the Proposition hold for the cohomology of complete intersections; the only non trivial point is the inequality $d \leq b^{+}$. 
We will use the notations of [D1]. Let $\mathbf{d}=\left(d_{1}, \ldots, d_{c}\right)$ be a sequence of positive integers. We denote by $V_{n}(\mathbf{d})$ a smooth complete intersection of multidegree $\mathbf{d}$ in $\mathbf{P}^{n+c}$. We put

$$
h^{p, q}(\mathbf{d})=\operatorname{dim} H^{p, q}\left(V_{p+q}(\mathbf{d})\right) \quad \text { and } \quad h_{\mathrm{o}}^{p, q}(\mathbf{d})=h^{p, q}(\mathbf{d})-\delta_{p, q} .
$$

Lemma 2.1. $h^{p+1, q+1}(\mathbf{d}) \geq h^{p, q}(\mathbf{d})$.

Proof : Following [D1] we introduce the formal generating series

$$
H(\mathbf{d})=\sum_{p, q \geq 0} h_{\mathrm{o}}^{p, q}(\mathbf{d}) y^{p} z^{q} \in \mathbf{Z}[[y, z]] ;
$$

we define a partial order on $\mathbf{Z}[[y, z]]$ by writing $P \geq Q$ if $P-Q$ has non-negative coefficients. The assertion of the lemma is equivalent to $H(\mathbf{d}) \geq y z H(\mathbf{d})$. The set $\mathcal{P}$ of formal series in $\mathbf{Z}[[y, z]]$ with this property is stable under addition and multiplication by any $P \geq 0$ in $\mathbf{Z}[[y, z]]$. The formula

$$
H\left(d_{1}, \ldots, d_{c}\right)=\sum_{\substack{P \subset[1, d] \\ P \neq \varnothing}}[(1+y)(1+z)]^{|P|-1} \prod_{i \in P} H\left(d_{i}\right)
$$

([D1], Cor. 2.4 (ii)) shows that it is enough to prove that $H(d)$ is in $\mathcal{P}$.

By [D1], Cor. 2.4 (i), we have $H(d)=\frac{P}{1-Q}$ with

$$
P(y, z)=\sum_{i, j \geq 0}\left(\begin{array}{c}
d-1 \\
i+j+1
\end{array}\right) y^{i} z^{j} \quad \text { and } \quad Q(y, z)=\sum_{i, j \geq 1}\left(\begin{array}{c}
d \\
i+j
\end{array}\right) y^{i} z^{j}
$$

Since $Q \geq y z$, we get $\frac{1-y z}{1-Q}=1+\frac{Q-y z}{1-Q} \geq 0$, hence $(1-y z) H \geq 0$.

Lemma 2.2. Let $d=d_{1} \ldots d_{c}$. We have:

a) $h^{p, p}(\mathbf{d}) \geq d$;

b) $2 h^{p+1, p-1}(\mathbf{d})+1 \geq d$, except in the following cases:

- $\mathbf{d}=(2),(2,2)$;

- $p=1, \mathbf{d}=(3),(2,3),(2,2,2),(2,2,2,2)$;

- $p=2, \mathbf{d}=(2,2,2)$.

Proof : We first prove b) in the case $p=1$. Then $V_{2}(\mathbf{d})$ is a surface $S$. The canonical bundle $K_{S}$ is $\mathcal{O}_{S}(e)$, with $e:=d_{1}+\ldots d_{c}-c-3$; therefore $K_{S}^{2}=e^{2} d$. The case $e \leq 0$ is immediate, so we assume $e \geq 1$. 
Then the index $K_{S}^{2}-8 \chi\left(\mathcal{O}_{S}\right)$ of the intersection form is negative $[\mathrm{P}$; if $e \geq 2$ we get $\chi\left(\mathcal{O}_{S}\right)>\frac{d}{2}$, hence $2 h^{2,0}(\mathbf{d})+1 \geq d$.

If $e=1$, we have $K_{S}=\mathcal{O}_{S}(1)$ hence $p_{g}=c+3$. The possibilities for $\mathbf{d}$ are $(5),(2,4),(3,3),(2,2,3)$ and $(2,2,2,2)$, and we have $2(c+3)+1 \geq d$ in each case except the last one. This also holds for $\mathbf{d}=(4)$, and the other cases are excluded.

Since the index is negative, we have $h^{1,1}(\mathbf{d})>2 h^{2,0}(\mathbf{d})+1$; this implies that a) holds (for $p=1$ ) except perhaps for $\mathbf{d}=(3),(2,2),(2,3)$, $(2,2,2)$. But the corresponding $h^{1,1}$ is $7,6,19,19$, which is always $>d$.

Now assume $p \geq 2$. a) follows from the previous case and Lemma 2.1, similarly it suffices to check b) for the values of $\mathbf{d}$ excluded in the case $p=1$. Using the above formulas we find

$$
h^{3,1}(3)=1, h^{3,1}(2,3)=8, h^{3,1}(2,2,2,2)=27, h^{4,2}(2,2,2)=6,
$$

so that $2 h^{p+1, p-1}(\mathbf{d})+1 \geq d$ for $p \geq 2$ in the three first cases and for $p \geq 3$ in the last one.

Theorem 2.3. Let $X$ be a smooth even-dimensional complete intersection in $\mathbf{P}^{n+c}$, of multidegree $\mathbf{d}=\left(d_{1}, \ldots, d_{c}\right)$. Let $d:=d_{1} \ldots d_{c}$ be the degree of $X$, and let e be the number of integers $d_{i}$ which are even.

Let $\left(b^{+}, b^{-}\right)$be the signature of the intersection form on $H^{n}(X, \mathbf{Z})$; we put

$$
s=b^{+}-b^{-} \quad, \quad t=\min \left(b^{+}, b^{-}\right) \quad, \quad u=\min \left(b^{+}-d, b^{-}\right) .
$$

We assume $\mathbf{d} \neq(2,2)$, and $\mathbf{d} \neq(3),(2,2,2,2)$ when $n=2$. Then:

- $H^{n}(X, \mathbf{Z})_{\mathrm{o}}=\langle-d\rangle \stackrel{+}{\oplus} \frac{s}{8} E_{8} \stackrel{\perp}{\oplus}(t-1) U$ if $\left(\begin{array}{c}\frac{n}{2}+e \\ e\end{array}\right)$ is even;

- $H^{n}(X, \mathbf{Z})_{\mathrm{o}}=A_{d-1} \stackrel{\perp}{\oplus} \frac{s-d}{8} E_{8} \stackrel{\perp}{\oplus} u U$ if $\left(\begin{array}{c}\frac{n}{2}+e \\ e\end{array}\right)$ is odd.

For a hypersurface, for instance, we find a lattice of the form $A_{d-1} \stackrel{\perp}{\oplus} p E_{8} \stackrel{\perp}{\oplus} q U$ if and only if $d$ is odd, or $d$ is even and $n$ is divisible by 4 .

Proof : We apply Proposition 1.1 with $L=H^{n}(X, \mathbf{Z})$. We take for $h$ the class of a linear section of codimension $\frac{n}{2}$, so that $h^{2}=d$.

By [L-W], Thm. 2.1 and Cor. 2.2, we know that

- $h$ is primitive;

- $h^{\perp}$ is even;

- $L$ is even or odd according to the parity of $\left(\begin{array}{c}\frac{n}{2}+e \\ e\end{array}\right)$. 
To apply the Proposition we only need the inequalities $b^{+} \geq d$ and $b^{-} \geq 2$. Note that the statement of the theorem holds trivially for $\mathbf{d}=(2)$, so we may assume $d \geq 3$. Let us write $n=4 k+2 \varepsilon$, with $\varepsilon \in\{0,1\}$. By Hodge theory we have

$$
b^{+}=\sum_{\substack{p+q=n \\ p \text { even }}} h^{p, q}+\varepsilon \quad b^{-}=\sum_{\substack{p+q=n \\ p \text { odd }}} h^{p, q}-\varepsilon ;
$$

when the inequalities a) and b) of Lemma 2.2 hold this implies $b^{+} \geq d$ and $b^{-} \geq 2$, so Proposition 1.1 gives the result.

In the exceptional cases of Lemma $2.2 \mathrm{~b}$ ), the lattice $L$ is even and we have $b^{+}, b^{-} \geq 2$, so Proposition 1.1 still applies.

Remark 2.4. The two first exceptions mentioned in the theorem are well-known ([D2], Prop. 5.2): we have $H^{2}(X, \mathbf{Z})_{\mathrm{o}}=E_{6}$ for a cubic surface, and $H^{n}(X, \mathbf{Z})_{\mathrm{o}}=D_{n+3}$ for a $n$-dimensional intersection of two quadrics. For an intersection of 4 quadrics in $\mathbf{P}^{6}$, we have $d=16$, hence by Proposition 1.1

$$
H^{2}(X, \mathbf{Z})_{\mathrm{o}}=\langle-16\rangle \stackrel{\perp}{\oplus} 6 E_{8}(-1) \stackrel{\perp}{\oplus} 15 U .
$$

\section{REFERENCES}

[D1] P. Deligne: Cohomologie des intersections complètes. SGA7 II, Exp. XI. Lecture Notes in Math. 340, 39-61; Springer, Berlin, 1973.

[D2] P. Deligne: Le théorème de Noether. SGA7 II, Exp. XIX. Lecture Notes in Math. 340, 328-340; Springer, Berlin, 1973.

[L-W] A. Libgober, J. Wood: On the topological structure of even-dimensional complete intersections. Trans. Amer. Math. Soc. 267 (1981), no. 2, 637-660.

[P] U. Persson: An introduction to the geography of surfaces of general type. Algebraic geometry, Bowdoin, 1985, 195-218; Proc. Sympos. Pure Math. 46, I, AMS, Providence, 1987.

[S] J.-P. Serre: Cours d'arithmétique. PUF, Paris, 1977.

[W] C. T. C. Wall: On the orthogonal groups of unimodular quadratic forms. Math. Ann. 147 (1962), 328-338.

Laboratoire J.-A. Dieudonné, UMR 6621 du CNRS, Université de Nice, Parc Valrose, F-06108 Nice Cedex 2, France

E-mail address: arnaud.beauville@unice.fr 